Pacific

Journal of

Mathematics

EXTREMALS FOR A MOSER-JODEIT EXPONENTIAL INEQUALITY

Steve Hudson and Mark Leckband

Volume $206 \quad$ No. 1

September 2002 


\title{
EXTREMALS FOR A MOSER-JODEIT EXPONENTIAL INEQUALITY
}

\author{
Steve Hudson and Mark Leckband
}

\begin{abstract}
Trudinger and Moser, interested in certain nonlinear problems in differential geometry, showed that if $|\nabla u|^{q}$ is integrable on a bounded domain in $R^{n}$ with $q \geq n \geq 2$, then $u$ is exponentially integrable there. Symmetrization reduces the problem to a one-dimensional inequality, which Jodeit extended to $q>1$. Carleson and Chang proved that this inequality has extremals when $q \geq 2$ is an integer. Hence, so does the Moser-Trudinger inequality (with $q=n$ ).

This paper extends the result of Carleson and Chang to all real numbers $q>1$. An application and some related results involving noninteger $q$ are also discussed.
\end{abstract}

\section{Introduction.}

Let $\mathrm{D}$ be a bounded domain in $\mathbf{R}^{n}, n \geq 2$. Let $W^{n}(D)$ be the Sobolev space of functions $u$ supported in the closure of $D$ with gradient in $L^{n}(D)$. Trudinger [10] showed that for $u$ in the unit ball of $W^{n}$, there are constants $\alpha$ and $A$ (depending only on $n$ ) such that

$$
\int_{D} \exp \left(\alpha u^{\frac{n}{n-1}}\right) d x \leq A|D| \text {. }
$$

Moser [9] found the largest possible value of $\alpha$ by using symmetrization to reduce this to a one-dimensional problem. The integer $n$ can then be replaced by a real number $q \geq 2$. Jodeit [5] extended the result to $1<q<2$.

Theorem A (Jodeit, Moser). Let $1<q<\infty, 1 / p+1 / q=1$. Let $\omega$ be a function in $C^{1}[0, \infty)$ such that $\omega(0)=0$ and $\int|w \prime|^{q} \leq 1$. Then

$$
A(q)=\sup _{w} \int_{0}^{\infty} \exp \left(\omega^{p}(t)-t\right) d t
$$

Carleson and Chang [2] proved that this theorem has extremals for integers $q \geq 2$. Through symmetrization, this proves that the Trudinger-Moser theorem for $W^{n}(D)$ has extremals, at least when $D$ is a ball. Flucher [3] extended this to arbitrary smooth bounded domains in $\mathbf{R}^{n}$. Lin $[\mathbf{6}]$ did the same for $n \geq 3$. It is natural to ask whether Theorem A has extremals for general $q$. The main result of this paper is: 
Theorem B. Theorem A has extremals for all real numbers $q>1$.

The outline of the proof is similar to that in [2], especially when $q \geq 2$. In Section 1 we show that if no extremals exist, then $A(q)$ is less than an explicit constant $R(q)$. This requires new methods when $1<q<2$. One of the main ideas of [2] is linearization, in which the exponent $p$ is replaced by 1 , with controllable error for $1<p \leq 2$. So, it is not surprising that their proof (their inequality (23), for example) breaks down when $1<q<2$.

Section 2 provides a specific $\omega$ to show $A(q)>R(q)$. This part requires a different construction than in [2], but for a different reason. There is less slack: It appears that $A(q) \rightarrow R(q)$ as $q \rightarrow 1^{+}$(but we do not attempt a proof of this).

In a related paper, McLeod and Peletier [8] give a somewhat different proof of Theorems A and B for integer $q>1$. It differs especially in the first part, in showing $A(q) \leq R(q)$. It then refers to the $\omega$ in [2].

It is not clear whether Theorem B has important applications to functions on $\mathbf{R}^{n}$ (with $q \neq n$ ). But there are several related results that show it is reasonable to look at noninteger $q$. For example, in Section 3, we use Theorem B to generalize the results in [2] to $u \in W^{q}\left(B^{n}\right), 1<q \leq n$, with similar sharpness in $\alpha$. When $q<n$ this involves a weight.

Also, Theorem A is used by the authors in [4] to prove an inequality like Moser's for functions in the Lorentz-Sobolev space $W^{n, q}(D)$. It isn't clear whether Theorem B gives extremals for this problem, due to problems with symmetrization.

Adams [1] has extended the Moser-Trudinger theorem to higher-order derivatives, based on a generalization of Theorem A by Garcia. A very interesting question is whether the inequality of Adams, or Garcia, has extremals. Our methods seem promising in showing that Garcia's inequality has them, for some range of $q$.

\section{Section 1.}

This section contains the proof of Proposition 1 below, and follows the strategy in [2]. Mainly, the range $1<q<2$ requires a new approach. We will generally avoid duplication of [2], except that our construction in the next section (unlike the one in [2]) is based on this work. So several equations from [2] are included here for later reference.

Let $R(q)=1+\exp \{\psi(q)+\gamma\}$, where $\psi(q)$ is the psi function $\Gamma^{\prime}(q) / \Gamma(q)$ and $\gamma$ is the Euler constant. If $q=n$ is an integer, then $R(q)$ is the CarlesonChang constant $1+\exp \{1+1 / 2+\cdots+1 /(n-1)\} . A(q)$ is the constant of Theorem A.

Proposition 1. If Theorem A has no extremal, then $A(q) \leq R(q)$. 
The following notation and results will be used in the proof. Let $K_{q}$ be the space of continuous piecewise $C^{1}$ functions $\omega(t)$ on $[0, \infty)$ satisfying

$$
\omega(0)=0, \omega^{\prime}(t) \geq 0, \quad \text { and } \quad \int_{0}^{\infty}\left|\omega^{\prime}\right|^{q} \leq 1 .
$$

Roughly, these are the functions of Theorem A.

Let $\omega_{m}$ be a sequence in $K_{q}$ such that $\int_{0}^{\infty} \exp \left(\omega_{m}^{p}(t)-t\right) d t$ converges to $A(q)$ as $m \rightarrow \infty$. Assuming Theorem A has no extremal, the following conditions hold:

(a) For each $A>0, \int_{0}^{A}\left|\omega_{m}^{\prime}(t)\right|^{q} d t \rightarrow 0$ as $m \rightarrow \infty$.

(b) For $m$ large enough, there exists a point $a_{m}$ in $[1, \infty)$ such that $\left(\omega_{m}\left(a_{m}\right)\right)^{p}-a_{m}=-2 \log ^{+}\left(a_{m}\right)$. Moreover, if $a_{m}$ denotes the first such point, then $a_{m} \rightarrow \infty$ as $m \rightarrow \infty$.

(c) $\lim \sup _{m \rightarrow \infty} \int_{a_{m}}^{\infty} \exp \left(\omega_{m}^{p}(t)-t\right) d t \leq \exp (\psi(q)+\gamma)$.

(d) $\lim _{m \rightarrow \infty} \int_{0}^{a_{m}} \exp \left(\omega_{m}^{p}(t)-t\right) d t=1$.

Proposition 1 follows from (c) and (d). The proofs in [2] require only minor modifications except for part (c) for $1<q<2$, which begins with the following lemma.

Lemma 1.1. For $1<q<\infty, p=q /(q-1)$ and $\delta>0$, let $K_{\delta, q}$ be the space of continuous piecewise $C^{1}$ functions on $[0, \infty)$ satisfying $\phi(0)=0$, $\phi^{\prime}(t) \geq 0$ and $\int\left(\phi^{\prime}\right)^{q} \leq \delta$. Then for each $c>0$,

$$
\sup _{\phi \in K_{\delta, q}} \int_{0}^{\infty} \exp \{c \phi(t)-t\} d t<\exp \left\{(1 / p)^{q-1} c^{q} \delta / q\right\} R(q) .
$$

While the proof resembles that in [2], we list the modifications required for noninteger $q$, and also some formulas needed later. Inequality (3) has an extremal $\phi$ such that,

$$
c \phi^{\prime}(t)=p\left(1+B e^{t /(q-1)}\right)^{-1},
$$

where $B \geq 0$ is chosen so that

$$
c^{q} \delta=\int_{0}^{\infty}\left(c \phi^{\prime}(t)\right)^{q} d t
$$

It is also shown $(B+1) / B$ is the numerical value of the supremum of (3). Let

$$
\begin{aligned}
& \beta(t)=\left[1+1 /\left(B e^{t /(q-1)}\right)\right]^{-1}, \quad \text { so that } \\
& \phi(t)=(q / c)[\log (1+1 / B)+\log (\beta(t))] .
\end{aligned}
$$


For further reference, define for $B \geq 0$,

$$
\begin{aligned}
\varepsilon(q, B) & =\int_{B+1}^{\infty}(u-1)^{-1}\left[1 / u-1 / u^{q}\right] d u, \\
& =\sum_{k=1} \frac{1}{k(B+1)^{k}}-\frac{1}{(k+q-1)(B+1)^{k+q-1}} .
\end{aligned}
$$

After a change of variables, the right side of (5) is equal to

$$
\begin{aligned}
p^{q}(q-1) \int_{B}^{\infty} \frac{1}{(u-1) u^{q}} d u & =p^{q}(q-1) \sum_{k=1} \frac{1}{(k+q-1)(B+1)^{k+q-1}}, \\
& =p^{q}(q-1)[\log (1+1 / B)-\varepsilon(q, B)], \\
& >p^{q}(q-1)[\log (1+1 / B)-(\psi(q)+\gamma)],
\end{aligned}
$$

where $\psi(q)+\gamma=\varepsilon(q, 0)>\varepsilon(q, B)$ for all $B>0$. Solving for $(B+1) / B$ in the above inequality establishes (3).

With the help of (7) and (8), the following extends a lemma of CarlesonChang to noninteger $q \geq 2$, which is used to prove (c) in this case.

Lemma 1.2.a. Let $\omega \in K_{q}$ and $\int_{a}^{\infty}\left(\omega^{\prime}\right)^{q}=\delta$. For $2 \leq q<\infty$ and $a>0$, we have

$$
\int_{a}^{\infty} \exp \left(\omega^{p}(t)-t\right) d t \leq \frac{\exp \left(\omega^{p}(a)-a\right)}{1-\delta^{\frac{1}{q-1}}} \exp \left(\frac{C_{1}^{q} \beta_{q}}{p^{q-1} q}\right) R(q),
$$

where $\beta_{q}=\delta /\left(1-\delta^{1 /(q-1)}\right)^{q-1}$ and $C_{1}=p \omega^{p-1}(a)$.

Our proof of (c) for $1<q<2$ requires a similar lemma:

Lemma 1.2.b. For $1<q<2$ and a large enough with $\omega^{p}(a)-a=$ $-2 \log (a)$, we have

$$
\begin{aligned}
& \int_{a}^{\infty} \exp \left(\omega^{p}(t)-t\right) d t \\
& \leq \frac{\exp \left(\omega^{p}(a)-a\right)}{1-\delta^{\frac{1}{q-1}}} \exp \left(\frac{C_{1}^{q}(1+\alpha)^{q} \beta_{q}}{p^{q-1} q}\right) R(q)+2 \exp (-a),
\end{aligned}
$$

where $\alpha=C_{2}(\log (a) / a)^{1 / q}$, for some constant $C_{2}$ independent of a. Proof of (10). For $a>1$, set $x=t-a, \psi(x)=\omega(t)-\omega(a)$. Then,

$$
(\omega(a)+\psi(x))^{p}=\omega^{p}(a)+p \omega^{p-1}[1+f(\psi(x) / \omega(a))] \psi(x)+\psi^{p}(x),
$$

where the function $\mathrm{f}$ comes from the binomial expansion of $(1+u)^{q}$. Note that $f$ is an increasing function and $f$ is $O(x)$ as $x \rightarrow 0$.

We have $\omega^{p}(a)-a=-2 \log (a)$ and $\omega^{p}(a) \leq a(1-\delta)^{\frac{1}{q-1}}$ This shows,

$$
\begin{aligned}
\delta & \leq 2(q-1) \frac{\log (a)}{a}+C \log ^{2}(a) / a^{2}, \quad\left(C \leq(2-q) 2^{2-q}\right) \\
& \leq C_{1} \log (a) / a .
\end{aligned}
$$


Let $E_{1}$ be the set of $x$ for which

$$
\psi(x) \geq 4 \omega(a)\left(\frac{C_{1} \log (a)}{a}\right)^{1 / q} .
$$

Then on $E_{1}$, using Holder's inequality and (12),

$$
\begin{aligned}
\omega(t) & \leq \psi(x)\left[1+\frac{1}{4}\left(\frac{a}{C_{1} \log (a)}\right)^{1 / q}\right] \\
& \leq \delta^{1 / q} x^{1 / p}\left[1+\frac{1}{4}\left(C_{1} \log (a)\right)^{1 / q}\right] \\
& \leq x^{1 / p}\left[\left(\frac{C_{1} \log (a)}{a}\right)^{1 / q}+\frac{1}{4}\right]
\end{aligned}
$$

We now require $a$ to be large enough so that $C_{1} \log (a) / a<1 / 4^{q}$. The integral of $\exp \left\{\omega^{p}-t\right\}$ over $E_{1}$ is bounded by,

$$
\int_{a}^{\infty} \exp \left(\frac{t-a}{2}-t\right) d t \leq 2 e^{-a}
$$

Let $E_{2}=\left\{x: \psi(x) \leq 4 \omega(a)\left(\frac{C_{1} \log (a)}{a}\right)^{1 / q}\right\}$. Replacing $\omega^{p}(t)$ by the right side of (11), we need to estimate the following integral,

$$
\int_{E_{2}} \exp \left(\omega^{p}(a)+p \omega^{p-1}(a)(1+f(\psi(x) / \omega(a))) \psi(x)+\psi^{p}(x)-x-a\right) d x .
$$

Using $\psi^{p}(x) \leq \delta^{1 /(q-1)} x$, we set

$$
y=\left(1-\delta^{1 /(q-1)}\right) x, \quad c_{1}=p \omega^{p-1}(a), \quad \phi(y)=\psi(x),
$$

and

$$
\alpha=C_{2}\left(\frac{\log (a)}{a}\right)^{1 / q} \geq f\left(4\left(\frac{C_{1} \log (a)}{a}\right)^{1 / q}\right),
$$

for some independent constant $C_{2}$. Observe that the previous integral is less than the following

$$
\frac{\exp \left(\omega^{p}(a)-a\right)}{1-\delta^{\frac{1}{q-1}}} \sup \int_{0}^{\infty} \exp \left(c_{1}(1+\alpha) \phi(y)-y\right) d y
$$

where the supremum is taken over all $\phi$ satisfying

$$
\int_{0}^{\infty}\left(\phi^{\prime}(y)\right)^{q} d y \leq \beta_{q} .
$$

We have the following inequality from (13) and (14).

$$
\int_{a}^{\infty} \exp \left(\omega^{p}(t)-t\right) d t \leq \frac{\exp \left(\omega^{p}(a)-a\right)}{1-\delta^{\frac{1}{q-1}}} \sup \int_{0}^{\infty} \exp (c \phi(y)-y) d y+2 e^{-a}
$$


where $c=(1+\alpha) c_{1}$. We now apply Lemma 1.1 proving (10).

We now return to the proof of (c) for $1<q<2$. The conclusion of Lemma 1.2.b implies

$$
\int_{a_{m}}^{\infty} \exp \left(\omega_{m}^{p}(t)-t\right) d t \leq \frac{e^{K} \exp (\psi(q)+\gamma)}{1-\delta_{m}^{1 /(q-1)}}+2 \exp \left(-a_{m}\right),
$$

where

$$
K=\omega_{m}^{p}\left(a_{m}\right)-a_{m}+\beta_{q}\left[p \omega^{p-1}\left(a_{m}\right)\left(1+C_{2} \log \left(a_{m}\right) / a_{m}\right)\right]^{q} /\left(p^{q-1} q\right) .
$$

All we need to show is that $\lim \sup K \leq 0$ as $m \rightarrow \infty$. The above expression for $K$ reduces to

$$
K=\omega_{m}^{p}\left(a_{m}\right)-a_{m}+\frac{\delta_{m}(1+\alpha)^{q}\left(\omega_{m}\left(a_{m}\right)\right)^{p}}{(q-1)\left(1-\delta_{m}^{1 /(q-1)}\right)^{q-1}} .
$$

We have $\left(\omega_{m}\left(a_{m}\right)\right)^{p}-a_{m}=-2 \log \left(a_{m}\right)$. Applying a binomial expansion to the denominator with estimate (12) derives

$$
K \leq-2 \log \left(a_{m}\right)+2 \log \left(a_{m}\right)+C\left(\log \left(a_{m}\right) / a_{m}\right)^{1+1 / q} .
$$

Observing $a_{m} \rightarrow \infty$ as $m \rightarrow \infty$ completes the proof of (c).

\section{Section 2.}

Here we prove that $A(q)>R(q)$ by studying specific examples $\omega_{q}$. Combined with Proposition 1 from Section 1, this proves Theorem B. When $q \geq 2$, we can use the Carleson-Chang example, but not their proof which uses induction on $q=n$. When $1<q<2$, we will need a new type of example, motivated by Section 1 , and some very precise estimates to show $A(q)>$ $R(q)$. In passing, it seems likely that $A(q)-R(q) \rightarrow 0$ as $q \rightarrow 1$.

Case 1. Suppose $2 \leq q<\infty$. Set

$$
\omega_{q}(t)= \begin{cases}{\left[(q-1)^{-1 / q} / p\right] t,} & 0 \leq t \leq q, \\ (t-1)^{1 / p}, & q \leq t \leq N_{q}, \\ \left(N_{q}-1\right)^{1 / p}, & t \geq N_{q},\end{cases}
$$

where $N_{q}=(q-1) \exp \left(p^{q}-p\right)+1$ is chosen so that $\int_{0}^{\infty}\left|\omega^{\prime}(t)\right|^{q} d t=1$.

One computes the exponential norm as the following:

$$
\begin{aligned}
I(q) & =\int_{0}^{\infty} \exp \left(\omega_{m}^{p}(t)-t\right) d t \\
& =q \int_{0}^{1} e^{v(t)} d t+(2-q) / e+(q-1) \exp \left(p^{q}-p-1\right),
\end{aligned}
$$

where $v(x)=(q-1) x^{p}-q x$. We prove the following lemma and thereby establish Theorem B.

Lemma 2.1. $I(q)>R(q)$ for $q \geq 2$. 
Proof. We observe for $v(x)=(q-1) x^{p}-q x$ that $v(0)=0, v(1)=-1$, $v^{\prime}(x)=q\left(x^{p-1}-1\right), v^{\prime}(0)=-q, v^{\prime}(1)=0$ and $v^{\prime \prime}>0$. Thus, $v(x)>-q x$ on $(0,1 / q]$ and $v(x) \geq-1$ on $[1 / q, 1]$. We estimate

$$
\int_{0}^{1} \exp (v)>\int_{0}^{1 / q} \exp (-q x)+\int_{1 / q}^{1} \exp (-1)=(1+(q-2) / e) / q,
$$

or

$$
q \int_{0}^{1} \exp (v)+(2-q) / e>1
$$

To complete the proof, it is enough to show, for $q \geq 2$,

$$
(q-1) \exp \left(p^{q}-p-1\right) \geq R(q)-1=\exp (\psi(q)+\gamma) .
$$

Both sides are equal to $e$ for $q=2$. For $q>2$, the problem reduces to showing

$$
\psi^{\prime}(q) \leq d / d q\left[p^{q}-p+\log (q-1)\right] .
$$

We now estimate both sides of (16). Observe,

$$
\begin{gathered}
\psi^{\prime}(q)=\sum_{k=0}^{\infty} \frac{1}{(k+q)^{2}} \leq \sum_{k=0}^{\infty} \frac{1}{\left(k+q-\frac{1}{2}\right)\left(k+q+\frac{1}{2}\right)} \\
=\frac{1}{\left(q-\frac{1}{2}\right)}, \\
d / d q\left(p^{q}-p\right)=p^{q}(\log (p)-p+1)+(p-1)^{2} .
\end{gathered}
$$

To prove (16), we must show

$$
\frac{1}{\left(q-\frac{1}{2}\right)} \leq(p-1)^{2}+(p-1)+p^{q}(\log (p)-p+1),
$$

which requires estimates of $\log (p)$ and $p^{q}$. Set $x=1 /(2 q-1) \leq 1 / 3$ so that $p=(1+x) /(1-x)$. A Maclaurin series expansion in $x$ shows that

$$
\frac{1}{\left(q-\frac{1}{2}\right)}+\frac{1}{12\left(q-\frac{1}{2}\right)^{3}} \leq \log (p) \leq \frac{1}{\left(q-\frac{1}{2}\right)}+\frac{1}{10\left(q-\frac{1}{2}\right)^{3}} .
$$

Therefore, from $d / d q\left[\log \left(p^{q}\right)\right]=\log (p)-1 /(q-1) \leq 0$, we have

$$
\log \left(p^{q}\right)=\log (4)+\int_{2}^{q} \log \left(\frac{t}{(t-1)}\right)-\frac{1}{(t-1)} d t,
$$

and using (17),

$$
\log \left(p^{q}\right) \leq \log (4)+\log ((2 q-1) / 3)+\varepsilon-\log (q-1),
$$

where

$$
\varepsilon=\int_{2}^{q} \frac{1}{\left(10\left(t-\frac{1}{2}\right)^{3}\right)} d t=(1 / 20)\left[\frac{4}{9}-\frac{1}{\left(q-\frac{1}{2}\right)^{2}}\right]<1 / 45 .
$$


So $p^{q} \leq 8(q-1 / 2) \exp (\varepsilon) /[3(q-1)]$. Since $\log (p)-p+1 \leq 0$, (16) has been reduced to proving

$$
\frac{1}{\left(q-\frac{1}{2}\right)} \leq \frac{1}{(q-1)}+\left(\frac{1}{(q-1)}\right)^{2}+\frac{8\left(q-\frac{1}{2}\right)}{3(q-1)} \exp (\varepsilon)\left(\log (p)-\frac{1}{(q-1)}\right) .
$$

Set $\lambda=p-1=1 /(q-1)$. Since

$$
\log (p)-\lambda \geq \frac{1}{\left(q-\frac{1}{2}\right)}-\frac{1}{(q-1)}=\frac{-1}{\left[2\left(q-\frac{1}{2}\right)(q-1)\right]},
$$

the right side of (18) is at least $\lambda+\lambda^{2}-(4 / 3) \lambda^{2} \exp \{\varepsilon\}$. The left side equals $2 \lambda /(\lambda+2)$, so (18) reduces to checking that

$$
[(4 / 3) \exp \{\varepsilon\}-1](\lambda+2) \leq 1 .
$$

If $q \geq 3$, (19) holds because $\varepsilon \leq 1 / 45$ and $\lambda \leq 1 / 2$.

Now suppose that $2<q \leq 3$. Since $\varepsilon \leq(q-2) / 30$, the mean value theorem shows that $\exp \{\varepsilon\} \leq 1+(q-2) / 28$. We also have $\lambda+2=(2 q-1) /(q-1)=$ $3-(q-2) /(q-1)$, so the left side of $(19)$ is at most

$$
\begin{gathered}
{[1 / 3+(q-2) / 21][3-(q-2) /(q-1)]} \\
\leq 1+(q-2)(1 / 7-1 /[3(q-1)]) \leq 1 .
\end{gathered}
$$

This completes the proof of Lemma 2.1.

Case 2. Suppose $1<q<2$.

Attempts to use examples like those in Case 1 indicate that the number of pieces required to beat $R(q)$ is unbounded as $q$ approaches 1 . We will construct an example that is linear over $[0, a]$ and nonlinear over $[a, \infty)$. We shall show that for large enough a, the exponential norm exceeds $R(q)$. In fact, as $a \rightarrow \infty$, the exponential norm of our example converges downward to $R(q)$. This is sufficient to establish the conclusion of Proposition 1 as false thereby proving Theorem B. The idea of how to do this is based upon the method of proof of Proposition 1 for $1<q<2$.

To begin, let $a>1$ and $\omega$ be linear on $[0, a]$ satisfying $\omega(0)=0$ and $\omega^{p}(a)-a=-2 \log (a)$. Define $\delta$ by the following:

$$
1-\delta=\int_{0}^{a}\left|\omega^{\prime}(t)\right|^{q} d t=\left(w^{p}(a) / a\right)^{q-1} .
$$

We look to Lemma 1.1 for the definition of our example over $[a, \infty)$. Recall that there is an explicit formula for an extremal for the supremum of (3). We use this formula below. For $t>a$, we define $x=t-a$ and $\omega(t)=\psi(x)+\omega(a)$, 
where

$$
\psi(x)=(q-1) A_{1}[\log (1+1 / B)+\log (\beta(x))], \text { and } \int_{0}^{\infty}\left(\psi^{\prime}(x)\right)^{q} d x \leq \delta .
$$

For ease of notation we have set

$$
\beta(x)=\left[1+1 /\left(B e^{\frac{x}{q-1}}\right)\right]^{-1} .
$$

We shall specify the constants $A_{1}$ and $B$ later.

The first estimate of the exponential integral is obvious.

$$
\int_{0}^{a} \exp \left(w^{p}(t)-t\right) d t>1-e^{-a} .
$$

The hard work is estimating the exponential integral over $[a, \infty)$.

The basic idea of [2] was to linearize the $(\omega(a)+\psi(x))^{p}$ and we do the same. However, the obvious inequality

$$
(\omega(a)+\psi(x))^{p} \geq \omega^{p}(a)+p \omega^{p-1}(a) \psi(x)+\psi^{p}(x),
$$

is too generous for our purposes. Therefore we expand as follows:

$$
\omega^{p}(t)=(\omega(a)+\psi(x))^{p}=\left(\mu+(q-1) A_{1} \log (\beta(x))\right)^{p},
$$

where $\mu=\omega(a)+(q-1) A_{1} \log (1+1 / B)=\omega(a)+\psi(\infty)$, to obtain

$$
\begin{aligned}
(\omega(a)+\psi(x))^{p}= & \mu^{p}+p \mu^{p-1}(q-1) A_{1} \log (\beta(x)) \\
& +(1 / 2) p(p-1)\left((q-1) A_{1}\right)^{2} \mu^{p-2} \log ^{2}(\beta(x)) \\
& +(1 / 6) p(p-1)(p-2)\left((q-1) A_{1}\right)^{3}(\zeta)^{p-3} \log ^{3}(\beta(x)),
\end{aligned}
$$

(where $\omega(a) \leq \zeta \leq \mu)$,

$$
\geq A_{2}+A_{3} \psi(x)+A_{4} \log ^{2}(\beta(x))+A_{5} \log ^{3}(\beta(x)),
$$

where

$$
\begin{aligned}
& A_{2}=\left(\omega(a)+(q-1) A_{1} \log (1+1 / B)\right)^{p-1}\left(\omega(a)-q A_{1} \log (1+1 / B)\right) \\
& \left(\text { or } A_{2}=\mu^{p-1}[\omega(a)-(p-1) \psi(\infty)]\right) \\
& A_{3}=p\left(\omega(a)+(q-1) A_{1} \log (1+1 / B)\right)^{p-1} \\
& A_{4}=(1 / 2) p(p-1)\left((q-1) A_{1}\right)^{2}\left(\omega(a)+(q-1) A_{1} \log (1+1 / B)\right)^{p-2}, \\
& A_{5}=(1 / 6) p(p-1)(p-2)\left((q-1) A_{1}\right)^{3} W \\
& \text { where } W= \begin{cases}(\omega(a))^{p-3}, & 2<p<3 . \\
(\omega(a)+\psi(\infty))^{p-3}, & 3 \leq p .\end{cases}
\end{aligned}
$$


We can now specify $A_{1}$ and $B$. Motivated by Equation (4), with the intention of having $c=A_{3}=p / A_{1}$, we want $A_{1}$ and $B$ to satisfy

$$
\begin{aligned}
A_{3} \psi^{\prime}(x) & =p\left(1+B e^{x /(q-1)}\right)^{-1}, \text { or equivalently, } \\
A_{1} & =\left(\omega(a)+(q-1) A_{1} \log (1+1 / B)\right)^{1-p}=(\mu)^{1-p} .
\end{aligned}
$$

And also, from (5), we want

$$
\frac{\delta}{A_{1}^{q}}=(q-1) \int_{B+1}^{\infty} \frac{d s}{(s-1) s^{q}} .
$$

It is not yet clear that there exist simultaneous solutions $A_{1}$ and $B$. To see this, let (25) define $A_{1}$ as a function of $B$. Define $L(B)$ as the left side and $R(B)$ as the right side of $(*)$. As $B \rightarrow \infty, A_{1} \rightarrow \omega(a)^{p-1}$ by (25), and $R(B) \rightarrow 0$. So, $L>R$ for large enough $B$. We compute,

$$
d R / d B=-(q-1) /\left[B(B+1)^{q}\right] \text { and } d L / d B=-\delta(q-1) /[B(B+1)] .
$$

Estimating the integrals as $B \rightarrow 0^{+}$shows $L<R$ for small enough $B$.

Note that (24) and (25) imply $A_{1}=p / A_{3}$. Setting $c=A_{3}$ in (3) implies that the extremal $\phi(x)$ for Lemma 1.1 is the $\psi(x)$ defined by (21). Thus, $\psi$ satisfies all the formulas in Lemma 1.1. We can now use (23) to proceed with the proof of Theorem B. The terms involving $\beta$ below were neglected error terms in [2], but contribute to an important 'good' integral $G$ defined below. The analysis is very tight.

We now have the following estimate:

$$
\begin{aligned}
& \int_{a}^{\infty} \exp \left(\omega^{p}(t)-t\right) d t \\
& \geq \exp \left(A_{2}-a\right) \int_{0}^{\infty} \exp \left(A_{3} \psi(x)+A_{4} \log ^{2}(\beta(x))+A_{5} \log ^{3}(\beta(x))-x\right) d x \\
& =\exp \left(A_{2}-a\right) \int \exp (\nu(x)+\eta(x)) d x \\
& =\exp \left(A_{2}-a\right)\left[\int \exp (\nu(x)) d x+G\right]
\end{aligned}
$$

where $\nu(x)=A_{3} \psi(x)-x, \eta(x)=A_{4} \log ^{2}(\beta(x))+A_{5} \log ^{3}(\beta(x))$ and

$$
G=\int_{0}^{\infty} \exp (\nu(x)+\eta(x))-\exp (\nu(x)) d x .
$$

We will be done if we show the right-hand side of (26) is larger than $R(q)-1+e^{-a}=\exp (\varepsilon(q, 0))+e^{-a}$. We now expand the right side of (26) into quantities that we must estimate. Since we have chosen $\psi$ to be an extremal, all the estimates of Lemma 1.1 will apply. In particular, we 
shall need the following identities to establish the estimates that have been organized into Lemmas 2.2 and 2.3.

(i) $a=\omega^{p}(a)(1-\delta)^{1-p}, \quad($ by $(20))$;

(ii) $(B+1) / B=\exp \left(\delta \mu^{p} /(q-1)+\varepsilon(q, B)\right)$, (by (8) and (25));

(iii) $\delta \mu=\psi(\infty)-\frac{(q-1) \varepsilon(q, b)}{\mu^{p-1}}$ (rearranging (ii));

(iv) $\exp \left(A_{2}-a\right) \int \exp (\nu(x))=\exp \left(A_{2}-a+\delta \mu^{p} /(q-1)\right) \exp (\varepsilon(q, B))$; ((iv) is equivalent to (ii)).

\section{Lemma 2.2.}

(a) $A_{2}-a+\delta \mu^{p} /(q-1) \geq-\varepsilon^{2}(q, B) /\left[\omega(a) \mu^{p-1}\right]$.

(b) $\varepsilon(q, 0)-\varepsilon(q, B) \leq(q-1) B$.

(c) $\varepsilon(q, B) \leq\left(\pi^{2} / 6-1 / p\right)(q-1)$.

Proof of (a). We begin with expanding the left side of (a) and simplifying using the definitions of $A_{2}, \mu, \omega(a)$ and $\psi(\infty)$, see (24).

$$
\begin{aligned}
& A_{2}-a+\delta \mu^{p} /(q-1) \\
& =\mu^{p-1}(\omega(a)-(p-1) \psi(\infty))-a+\frac{\delta \mu^{p}}{(q-1)}, \\
& =-a+\mu^{p-1}\left[\omega(a)-(p-1) \psi(\infty)+\frac{\delta \mu}{(q-1)}\right],
\end{aligned}
$$

using $-(p-1)+1 /(q-1)=0$ and the right side of (27iii) for $\delta \mu$,

$$
\begin{aligned}
& =-a+\mu^{p-1}\left[\omega(a)-\varepsilon(q, B) \mu^{1-p}\right], \\
& =-a+\omega^{p}(a)[1+\psi(\infty) / \omega(a)]^{p-1}-\varepsilon(q, B),
\end{aligned}
$$

using (27iii) to solve for $\psi(\infty) / \omega(a)$,

$$
=-a-\varepsilon(q, B)+\omega^{p}(a)\left[\frac{1}{1-\delta}+\frac{(q-1) \varepsilon(q, B)^{p-1}}{(1-\delta) \omega(a) \mu^{p-1}}\right]^{p-1} .
$$

For $p \geq 2,(x+y)^{p-1} \geq x^{p-1}+(p-1) x^{p-2} y$ and the above reduces to $(28)$

$$
\geq-a-\varepsilon(q, B)+\omega^{p}(a)(1-\delta)^{1-p}+\varepsilon(q, B)[(1-\delta)(1+\psi(\infty) / \omega(a))]^{1-p} .
$$

Using (27i) and the following version of (27iii),

$$
(1-\delta)(1+\psi(\infty) / \omega(a))=1+\frac{(q-1) \varepsilon(q, B)}{\omega(a)(\omega(a)+\psi(\infty))^{p-1}} .
$$


The right side of $(28)$ is

$$
\begin{aligned}
& =-\varepsilon(q, B)+\varepsilon(q, B)\left[1+(q-1) \varepsilon(q, B) /\left(\omega(a) \mu^{p-1}\right)\right]^{1-p}, \\
& \geq-\varepsilon(q, B)+\varepsilon(q, B)\left[1-\varepsilon(q, B) /\left(\omega(a) \mu^{p-1}\right)\right]
\end{aligned}
$$

which completes the Proof of (a).

Proof of (b). By (7) we have

$$
\begin{aligned}
\varepsilon(q, 0)-\varepsilon(q, B) & =\int_{1}^{B+1} \frac{1}{(s-1)}\left[\frac{1}{s}-\frac{1}{s^{q}}\right] d s \\
& \leq \int_{1}^{B+1}(q-1) / s d s \leq(q-1) B .
\end{aligned}
$$

Proof of (c). Using the series representation of $\varepsilon(q, 0)$, see $(7)$,

$$
\begin{aligned}
\varepsilon(q, B) \leq \varepsilon(q, 0) & =\sum_{k=1}^{\infty}\left(\frac{1}{k}-\frac{1}{k+q-1}\right), \\
& =(q-1)\left(1+\sum_{k=2}^{\infty} \frac{1}{k(k+q-1)}-\frac{1}{p},\right) \\
& \leq(q-1)\left(\sum_{k=1}^{\infty} \frac{1}{k^{2}}-\frac{1}{p}\right), \text { which is (c). }
\end{aligned}
$$

Lemma 2.3. For large enough $a, G \geq q(q-1)[1-3 / a] /\left[B \mu^{p}\right]$.

Proof. By integration by parts,

$$
\int_{0}^{\infty} \exp (\nu(x)+\eta(x)) d x=V(\infty)-\int_{0}^{\infty} V(x) \exp (\eta(x)) \eta^{\prime}(x) d x,
$$

where

$$
V(x)=\int_{0}^{x} \exp (\nu(t)) d t
$$

and $\eta(x)=A_{4} \log ^{2}(\beta(x))+A_{5} \log ^{3}(\beta(x)), \beta(x)=\left[1+1 /\left(B e^{x / q-1}\right)\right]^{-1}$.

We need to explicitly calculate $V(x)$. To begin, $\nu(t)=A_{3} \psi(t)-t$, where $\psi$ is an extremal for (3). Therefore, a variational argument shows that $\nu$ satisfies,

$$
e^{\nu(t)}=A \nu^{\prime \prime}(t)\left(\nu^{\prime}(t)+1\right)^{q-2}, \text { for some constant } A<0 .
$$

Observe $\nu(0)=0, \nu^{\prime}(\infty)=-1$ and $\nu(\infty)=-\infty$. Multiply (29) by $\nu^{\prime}(t)$ and integrate to obtain,

$$
e^{\nu(t)}=\frac{A\left(\nu^{\prime}(t)+1\right)^{q}}{q}-\frac{A\left(\nu^{\prime}(t)+1\right)^{q-1}}{q-1}+C .
$$


Let $t \rightarrow \infty$ to obtain $C=0$. Equations (29) and (30) imply

$$
\nu^{\prime \prime}(t)=\frac{\left(\nu^{\prime}(t)+1\right)^{2}}{q}-\frac{\left(\nu^{\prime}(t)+1\right)}{q-1} .
$$

Solving this differential equation shows the following version of (4):

$$
\nu^{\prime}(t)+1=p\left(1+B e^{t /(q-1)}\right)^{-1} .
$$

It can be shown, see $[\mathbf{2}]$, that $V(\infty)=(B+1) / B=J=1 / \beta(0)$. Using (30) and (31) we compute,

$$
V(x)=-A p^{q-1} /(q-1)\left[(B+1)^{1-q}-(1-\beta(x))^{q-1}\right] .
$$

Using $V(\infty)=(B+1) / B$ and the above with $\beta(\infty)=1$, we have

$$
V(x)=(B+1)^{q} / B\left[(B+1)^{1-q}-(1-\beta(x))^{q-1}\right] .
$$

Notice $\beta \leq 1, \exp (\eta(x)) \geq 1$ and $\eta^{\prime}(x) \leq 0$, thus the above gives,

$$
G \geq 1 / B \int_{0}^{\infty}\left[(B+1)^{1-q}-(1-\beta(x))^{q-1}\right]\left|\eta^{\prime}(x)\right| d x,
$$

and setting $w=\log (\beta)$,

$$
=-1 / B \int_{-\log (J)}^{0}\left(2 A_{4} w-3 A_{5} w^{2}\right)\left[(B+1)^{1-q}-\left(1-e^{w}\right)^{q-1}\right] d w .
$$

Using a Maclaurin series representation for $(1-x)^{q-1}$ and $(B+1)^{-1}=$ $1-1 / J$

$$
G \geq 1 / B \int_{-\log (J)}^{0}\left(2 A_{4} w-3 A_{5} w^{2}\right)(q-1)\left[e^{w}-B /(B+1)\right] d w,
$$

integrating by parts and using the definitions of $A_{4}$ and $A_{5}$ (see (24)) gives,

$$
=\left[q(q-1) /\left(B \mu^{p}\right)\right]\left[1-(p-2)(q-1) / \mu^{p}+O\left(\log ^{3}(J) / J\right)\right] .
$$

Notice that, $\mu^{p} \approx \omega^{p}(a) \approx a$, as $a$ approaches $\infty$. By (27iii),

$$
\mu \geq \omega(a) /(1-\delta)=(a / \omega(a))^{q-1} .
$$

So $\mu^{p} \geq \mu^{p-1} \omega(a) \geq a$. By $(20), \delta \geq 2(q-1) \log (a) / a$. This and (27ii) give $B$ is $O\left(1 / a^{2}\right)$. Thus $\log ^{3}(J) / J$ is $o(1 / a)$, and so

$$
G \geq q(q-1)[1-3 / a] /\left(B \mu^{p}\right) .
$$

This completes the proof of Lemma 2.3. We return to the proof of Theorem B.

Using (22), (26), and (27iv),

$$
\begin{aligned}
& \int_{0}^{\infty} \exp \left(\omega^{p}(x)-x\right) d x \\
& >1-e^{-a}+\exp \left(A_{2}-a+\delta \mu^{p} /(q-1)\right) e^{\varepsilon(q, B)}+\exp \left(A_{2}-a\right) G .
\end{aligned}
$$


We shall show the right side is greater than or equal to $1+e^{\varepsilon(q, 0)}$. Using algebra and (27ii) this goal becomes,

$$
\exp \left(A_{2}-a+\delta \mu^{p} /(q-1)\right)[1+B G /(B+1)] \geq e^{-\varepsilon(q, B)}\left[e^{\varepsilon(q, 0)}+e^{-a}\right] .
$$

By Lemma 2.2(a) and Lemma 2.3, the left side of the above is greater than or equal to

$$
\exp \left(-\varepsilon^{2}(q, B) /\left(\omega(a) \mu^{p-1}\right)\right)\left[1+q(q-1)(1-3 / a) /\left[\mu^{p}(1+B)\right]\right],
$$

and,

$$
\varepsilon^{2}(q, B) \leq\left(\pi^{2} / 6-1 / p\right)^{2}(q-1)^{2}, \quad(\text { by Lemma } 2.2(\mathrm{c})) .
$$

Since $\pi^{2} / 6-1 / p<p$ for $p>2$ and $p(q-1)=q$, for large enough $a$ the above is

$$
\leq(q-\log (a) / a)(q-1) .
$$

We also claim $1 / a-1 / \mu^{p}$ is $O\left(1 / a^{2}\right)$. To see this, from (27i) and (27iii),

$$
\mu^{p} \leq(a / \omega(a))^{p}(1+(q-1) \varepsilon(q, B) / a)^{p} .
$$

A binomial expansion shows $(1+(q-1) \varepsilon(q, B) / a)^{p}$ is $1+O(1 / a)$. So,

$$
\begin{aligned}
\limsup _{a \rightarrow \infty}\left(\mu^{p}-a\right) & \leq \limsup _{a \rightarrow \infty}\left[(a / \omega(a))^{p}-a\right]+O(1) \\
& =O(1) .
\end{aligned}
$$

Therefore, $1 / a-1 / \mu^{p} \approx\left(\mu^{p}-a\right) / a^{2}$ is $O\left(1 / a^{2}\right)$.

Recall that $B$ is $O\left(1 / a^{2}\right)$, so the factor $B+1$ is negligible and the left side of $(33)$ is at least

$$
\begin{aligned}
& {[1-(q-\log (a) / a)(q-1) / a][1+q(q-1)(1-3 / a) / a]+O\left(1 / a^{2}\right),} \\
& \geq 1+(q-1) \log (a) / a^{2}+O\left(1 / a^{2}\right), \\
& \geq 1+(q-1)\left[B+(\log (a)-1) / a^{2}\right]+O\left(1 / a^{2}\right) .
\end{aligned}
$$

Lemma 2.2(b) implies the right side of (33) is at most $1+(q-1) B+O\left(1 / a^{4}\right)$, completing the proof.

\section{Section 3. An application of Theorem B.}

For real valued functions $f$ on $R^{n}$, let $f^{*}$ be the nonincreasing rearrangement of $f$ defined as $f^{*}(t)=\inf \{s: m\{|f|>s\} \leq t\}$. We define $f^{\#}(x)$ to be the spherically symmetric nondecreasing rearrangement of $\mathrm{f}$ defined as $f^{\#}(x)=f^{*}\left(\sigma_{n-1}|x|^{n} / n\right)$ where $\sigma_{n-1}$ is the $n-1$ measure of the unit sphere.

We have the following theorem which includes the case $q=n$ which is the application of Carleson and Chang, [2]. 
Theorem C. Let $1<q \leq n$. For functions $u$ supported in $B^{n}$ such that $\|\nabla u\|_{q} \leq 1$,

$$
\int_{B^{n}} \exp \left(\alpha u^{\# p}(x)\right) m(|x|) d x \leq A(q)\left|B^{n}\right|,
$$

where

$$
m(r)=\frac{\exp \left\{-\left(r^{-k n}-1\right)\right\}}{r^{-n(k+1)}}
$$

and

$$
k=\frac{(n-q)}{n(q-1)}, \alpha=n\left(\sigma_{n-1}\right)^{1 /(q-1)} .
$$

If $q=n$, set $m(r)=1$.

This is sharp in the sense that it does not hold for any larger $\alpha$. There is an extremal for each $1<q \leq n$. Also, $m(r)$ is continuous as a function of $q$.

By standard symmetrization, we can assume $u=u^{\#}$. Set $|x|=e^{-t / n}$, $v(t)=\alpha^{1 / p} u^{\#}(x)$ and note $\left|v^{\prime}(t)\right|=\left(\alpha^{1 / p}|x| / n\right)\left|\nabla u^{\#}(x)\right|, d x=-\left|B^{n}\right| e^{-t} d t$. So,

$$
\int_{0}^{\infty}\left|v^{\prime}(t)\right|^{q} e^{t(q-n) / n} d t \leq 1
$$

For $1<q<n$, set $t=\ln (k s+1) / k$, so $s=\left(e^{k t-1}\right) / k$. Set $\omega(s)=v(t)$. Then,

$$
\int_{0}^{\infty}\left|\omega^{\prime}(s)\right|^{q} d s \leq 1 .
$$

By Theorem A, $\int \exp \left(\omega^{p}(s)-s\right) d s \leq A(q)$, and this has an extremal by Theorem B. Thus,

$$
\int_{0}^{\infty} \exp \left(v^{p}(t)-s(t)\right) d s(t) \leq A(q)
$$

and this has an extremal. This is the conclusion of Theorem C.

\section{References}

[1] D.R. Adams, A sharp inequality of J. Moser for higher order derivatives, Annals of Math., 128 (1988), 385-398, MR 89i:46034, Zbl 9672.31008.

[2] L. Carleson and S.Y.A. Chang, On the existence of an extremal function for an inequality of J. Moser, Bull. Sc. Math., $2^{e}$ série, 110 (1986), 113-127, MR 88f:46070, Zbl 0619.58013.

[3] M. Flucher, Extremal functions for the Trudinger-Moser inequality in 2 dimensions, Comm. Math. Hel., 67 (1992), 471-497, MR 93k:58073, Zbl 0763.58008.

[4] S. Hudson and M. Leckband, A sharp exponential inequality for Lorentz-Sobolev spaces on bounded domains, Proc. Amer. Math. Soc., 127(7) (1999), 2029-2033, MR 99j:46036, Zbl 0920.46025. 
[5] M. Jodeit, An inequality for the indefinite integral of a function in $L^{q}$, Studia Math., 44 (1972), 545-554, MR 49 \#5805, Zbl 0244.26010.

[6] M.A. Leckband, An integral inequality with applications, Trans. Amer. Math. Soc., 283(1) (1984), 157-168, MR 85g:26018, Zbl 0545.26006.

[7] K.-C. Lin, Extremal functions for Moser's inequality, Trans. Amer. Math. Soc., 348(7) (1996), 2663-2671, MR 96i:58043, Zbl 0861.49001.

[8] J.B. McLeod and L.A. Peletier, Observations on Moser's inequality, Arch. Rat. Mech. Anal., 106 (1989), 261-285, MR 90d:26029, Zbl 0687.46017.

[9] J. Moser, A sharp form of an inequality by N. Trudinger, Ind. Univ. Math. J., 23 (1971), 1077-1092, MR 46 \#662, Zbl 0213.13001.

[10] N.S. Trudinger, On imbeddings into Orlicz spaces and some applications, J. Math. Mech., 17 (1967), 473-484, MR 35 \#7121, Zbl 0163.36402.

Received July 19, 1999.

Mathematics Department

Florida INTERNATIONAL UNIVERSITy

Miami, FL 33199

E-mail address: hudsons@fiu.edu

Mathematics Department

FLORIDA INTERNATIONAL UNIVERSITY

MiAMI, FL 33199

E-mail address: leckband@fiu.edu 